\title{
Feeding for Patients with Neurodegenerative Disoders as a Focus on Niemann Type C Pick Disease (Npd)
}

\author{
Copetti Santos $\mathrm{D}^{1 *}$ and Santos Gonçalves $\mathbf{N}^{2}$ \\ ${ }^{1}$ Department of Biological Sciences-biochemistry, Federal Institute of Education, Science and Technology Ragamuffin, Brazil \\ ${ }^{2}$ Student of the medicine course of the Southern Institute of Passo Fund, Rio Grande do Sul-Brazil
}

*Corresponding author: Daniela Copetti Santos, Department of Biological Sciences-biochemistry, Federal Institute of Education, Science and Technology Ragamuffin, Brazil

Submission: April 24, 2018; Published: June 01, 2018

\section{Introduction}

The brain has a remarkable capacity for plasticity, an aptitude that ironically contrasts with the limited therapeutic approaches that would reduce the consequences of brain insults. Although a large amount of clinical trials have been performed to test the efficacy of various pharmacological compounds in minimizing the burden of neurological disorders, the results of many of these trials have been unsuccessful.

An increasing number of basic science studies indicate that environmental conditions and experiences encountered in the daily routine of individuals can dramatically affect the capacity of the brain to react to challenges. Niemann Pick Type C disease (NPC) is a neurodegenerative disease with a recessive genetic inheritance and that the challenges encountered by the individual who has it both in its improvement and in its treatment may be directly associated with environmental conditions. It's a rare disease that leads to a change in the metabolism of cholesterol and other fats and, therefore, there is an accumulation of these substances in the body, especially in the liver, spleen and brain cells. We can say that the metabolic pathways of these individuals are like a congested transit of vehicles, while a person who does not have the disease has free and properly flowing traffic. Cells that suffer from the accumulation of substances stop functioning and lose their functions and are considered a deposit lysosomal disease caused by an inborn error of metabolism. Lipids do not accumulate only in the brain, but also in the liver and spleen causing a hepatosplenomegaly.

The NPC disease raises a number of doubts among parents, educators and caregivers in general. Because it is rare and requires a very specific diagnostic investigation, the syndrome is unknown to most people, so once the diagnosis is confirmed, it is normal for the people around the child to not know exactly what to do to help them, to have a better quality of life, within their means.
In our country there is the Niemann Pick Association-Brazil [1] and has the mission of disseminating information about the disease, aiming at early identification of cases so that therapeutic measures can be adopted, thus avoiding rapid progression and worsening of the disease. Created in December 2010, it results from a longing for the families of Brazilian carriers. Like all associations of rare disease patients, its mission is to guarantee the patients - in the three types of presentation A, B and C - access to medicines and treatments that provide them with better quality of life, demanding attention to patients' rights of hereditary genetic diseases by government institutions, as well as by health plans, as the case may be.

There is currently an approved therapy to decelerate the symptoms of the disease, which is the Substance Reduction Therapy, TRS, through the substance called Miglustate found in the drug Zavesca. Also for the treatment of NPC, in the research is Cyclodextrin that has proven, in the clinical study, performed in the United States, act alongside the Miglustate, very efficiently, in the stabilization and even improvement of some neurological symptoms such as muscle tone, dysphagia and cognition [1]. Allied to all this, it is important that the family is well-oriented as to the limitations and special care that this child requires, as well as in relation to their food. This issue can make a huge difference in the quality of life of this child. The constant problems of the broad neurodegenerative symptomatology of Type $\mathrm{C}$ are treated with palliative medications and care of physical therapy, speech therapy and nutrition. Medication, which is important in the treatment, brings side effects that can be minimized with an adequate diet and, in addition, the body of the child with NPC has limitations for the ingestion of certain substances, which can aggravate their condition. However, building a balanced, healthy and, above all, delicious diet for the child is possible, from simple exchanges and substitutions on the menu. 
In Portugal there is a Food Guide for NPC patients treated with miglustat [2], including in this guide there are several recipes tasty and easy to prepare for these patients. In general terms, it is recommended that the child undergoing treatment avoid all types of white pasta (white bread, pasta, cakes, etc.), sugary foods in general and foods containing lactose (practically all dairy products).

For example, whole grains (breads, noodles and cakes made with wholemeal flour), sweetened foods with sweeteners and soy-based drinks ("milk", yogurts and cheeses), for example, can be consumed in moderation. They are also in the food group that can be moderately administered to potatoes and brown rice, due to diarrhea that may be associated with these types of foods. The diarrhea resulting from lactose intolerance is due to the presence of unabsorbed carbohydrates, active osmotically and products that are fermented in the intestinal lumen, this being the main osmotic effect [3]. The syndrome of malabsorption with lactose is expressed as intolerance to it and depends on some other factors such as the maturation of the gastrointestinal tract, maturation of the activity of the disaccharidases, transport of monosaccharides, type of feeding Received either breast milk, infant formulas or cow milk, side effect of the drug (MIGLUSTAT) because it inhibits the activity of intestinal disaccharidases, food osmolarity and reclamation capacity of the colon, which It is responsible for the resorption of water, electrolytes and gases, all of these factors influencing the severity of diarrhea [4].

Due to the a fore mentioned, in this type of patients lactose intolerance is secondary to the administration of the drug (MIGLUSTAT), presenting as a clinical manifestation, recurrent episodes of diarrhea. To prevent this gastrointestinal disturbance and calcium deficiency, a lactose-free formula for children under 1year and $10 \mathrm{Kg}$ should be included in the nutritional management, or if it is not possible to continue with lactose-free formula for economic reasons, use milk Lactose for those older than 1 year and $10 \mathrm{Kg}$. In addition, it should be supplemented with calcium to ensure proper functioning of the cells, transmission of nerve impulse, osteogenesis and muscular contraction processes [5].

For these children, fruits (except bananas, peaches and dried fruit), eggs, nuts, nuts and seeds are usually released. In terms of meats, it is necessary to give preference to the lean ones and, of oils, to opt for the preparation with those of soy, safflower or margarine. It is worth mentioning that having a professional support network is fundamental to the care plan that the family will set up for the child with NPC. In addition to the clinical follow-up with the Child Neurologist, the composition of the daily menu can be made with the help of a Nutritionist, which will make the family safer. Even in the face of the limitations that the disease imposes, the child with NPC will certainly be more disposed and with fewer adverse effects, such as nausea and diarrhea, if their food is healthy, delicious and fun at the same time. With the right information, this is possible. There is also a booklet created by the Association NPC-Brazil, which shows that children with the disease as the symptoms become more pronounced should be submitted to a gastrostomy so that the feeding is done directly in the stomach. Attention should also be paid to hydration and weight control, because in some cases the disease can lead to malnutrition by a factors, including difficulty in swallowing. In these situations, food supplementation is required.

\section{A Way to Facilitate the Swallowing of Food for these Patients}

Because of the difficulty in swallowing, it is sometimes necessary to resort to artifacts such as wrapping the capsule or tablet in avocado or beaten strawberry, for example. In the case of patients with gastric tube the capsules and tablets should be water diluted for administration, in the same way as in the consistency of syrup or gel. It is always good to avoid, even in cases where dysphagia is not severe, intake of food that may cause gagging. Studies by Patterson et al. [6] show that swallowing assessments were done at screening and at months 6 and 12 . Patients were asked to swallow the following substances (in increasing level of difficulty): $5 \mathrm{~mL}$ of water, 1 teaspoon of purée, 1 teaspoon of soft lumps (tinned spaghetti or noodles), or a third of a cookie. Three attempts were made for each substance and the study assessment was done on the final attempt.

The assessor evaluated the patient's swallowing ability using a five-degree category scale: "no problems swallowing", "mild", "moderate", "severe", or "could not swallow the substance at all". Adverse events that patients presented throughout the disease as diarrhea $(17 / 20 ; 85 \%)$, flatulence $(14 / 20 ; 70 \%)$, Weight loss $(13 / 20 ; 65 \%)$, and abdominal pain $(10 / 20 ; 50 \%)$. The incidence of diarrhea, $\mathrm{fl}$ atulence, abdominal pain, vomiting and tremors decreased over time when they started using Miglustat.and the food they presented the most difficulty swallowing was the biscuits Disfagia y disartria progresivas: el habla y la deglución se alteran de forma progresiva y al final, la alimentación por vía oral se hace imposible. The difficulty occurs due to the loss of muscular tonus also alters the posture of the patient, Due to the nutritional problems that may appear due to bulbar involvement, it is essential to ensure correct caloric intake and adequate vitamin intake. In advanced stages of the disease, it is recommended the placement of a nasogastric tube or the introduction of a gastrostomy to avoid the risk of food aspirations and bronchopneumonia.

\section{References}

1. (2018) Brazil Niemann Pick Association, Brazil.

2. Actelion Pharmaceuticals Portugal (2018) Guia de Alimentação para Doentes com Niemann Pick tipo C tratados com Miglustato, Portugal.

3. Amorim S (2018) Feeding and quality of life of the child with NPC.

4. Niemann Pick Association (2018) What you need to know about Niemann Pick C, Brazil.

5. Soares J (1995) Calcium Bioavailability. Bioavailability of nutrients for animal's amino acids. Minerals and vitamins, Maryland, USA, pp. 95-100.

6. Patterson MC, Vecchio D, Prady H, Abel L, Wraith JE (2007) Miglustat for treatment of Niemann-Pick C disease: a randomised controlled study. Lancet Neurol 6(9): 765-772. 
Creative Commons Attribution 4.0 International License

For possible submissions Click Here

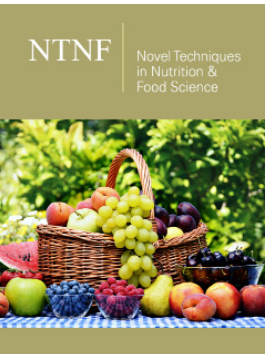

Novel Techniques in Nutrition and Food Science

\section{Benefits of Publishing with us}

- High-level peer review and editorial services

- Freely accessible online immediately upon publication

- Authors retain the copyright to their work

- Licensing it under a Creative Commons license

- Visibility through different online platforms 